\title{
Therapeutic suppression of translation initiation factor elF4E expression reduces tumor growth without toxicity
}

\author{
Jeremy R. Graff, ${ }^{1}$ Bruce W. Konicek, ${ }^{1}$ Thomas M. Vincent, ${ }^{2}$ Rebecca L. Lynch, ${ }^{1}$ David Monteith, ${ }^{1}$ \\ Spring N. Weir, ${ }^{1}$ Phil Schwier, ${ }^{1}$ Andrew Capen, ${ }^{1}$ Robin L. Goode, ${ }^{1}$ Michele S. Dowless, ${ }^{1}$ \\ Yuefeng Chen, ${ }^{1}$ Hong Zhang, ${ }^{2}$ Sean Sissons, ${ }^{1}$ Karen Cox, ${ }^{1}$ Ann M. McNulty, ${ }^{1}$ Stephen H. Parsons, ${ }^{1}$ \\ Tao Wang, ${ }^{1}$ Lillian Sams, ${ }^{1}$ Sandaruwan Geeganage, ${ }^{1}$ Larry E. Douglass, ${ }^{3}$ Blake Lee Neubauer, ${ }^{1}$ \\ Nicholas M. Dean, ${ }^{2}$ Kerry Blanchard, ${ }^{1}$ Jianyong Shou, ${ }^{1}$ Louis F. Stancato, ${ }^{1}$ \\ Julia H. Carter, ${ }^{3}$ and Eric G. Marcusson ${ }^{2}$
}

${ }^{1}$ Lilly Research Labs, Eli Lilly and Company, Indianapolis, Indiana, USA. ${ }^{2}$ Isis Pharmaceuticals, Carlsbad, California, USA. 3Wood Hudson Cancer Research Laboratory, Newport, Kentucky, USA.

\begin{abstract}
Expression of eukaryotic translation initiation factor 4E (eIF4E) is commonly elevated in human and experimental cancers, promoting angiogenesis and tumor growth. Elevated eIF4E levels selectively increase translation of growth factors important in malignancy (e.g., VEGF, cyclin D1) and is thereby an attractive anticancer therapeutic target. Yet to date, no eIF4E-specific therapy has been developed. Herein we report development of eIF4E-specific antisense oligonucleotides (ASOs) designed to have the necessary tissue stability and nuclease resistance required for systemic anticancer therapy. In mammalian cultured cells, these ASOs specifically targeted the eIF4E mRNA for destruction, repressing expression of eIF4E-regulated proteins (e.g., VEGF, cyclin D1, survivin, c-myc, Bcl-2), inducing apoptosis, and preventing endothelial cells from forming vessel-like structures. Most importantly, intravenous ASO administration selectively and significantly reduced eIF4E expression in human tumor xenografts, significantly suppressing tumor growth. Because these ASOs also target murine eIF4E, we assessed the impact of eIF4E reduction in normal tissues. Despite reducing eIF4E levels by $80 \%$ in mouse liver, eIF4E-specific ASO administration did not affect body weight, organ weight, or liver transaminase levels, thereby providing the first in vivo evidence that cancers may be more susceptible to eIF4E inhibition than normal tissues. These data have prompted eIF4E-specific ASO clinical trials for the treatment of human cancers.
\end{abstract}

\section{Introduction}

Eukaryotic translation initiation factor 4E (eIF4E) binds the $5^{\prime}$ cap structure of cellular mRNAs, delivering these mRNAs to the eIF4F translation initiation complex. This complex then scans $5^{\prime}-3^{\prime}$ from the cap, unwinding secondary structure in the mRNA $5^{\prime}$ untranslated region ( $\left.5^{\prime} \mathrm{UTR}\right)$ to expose the translation initiation codon, enable ribosome loading, and promote translation $(1,2)$. eIF4F complex assembly is rate limiting for initiation and dependent upon eIF4E availability. Under normal cellular conditions, eIF4E is bound by eIF4E binding proteins (4E-BPs), thereby limiting eIF4F complex assembly. Stimulation of the PI3K/AKT/mTOR pathway leads to hierarchical 4E-BP phosphorylation, eIF4E release, and eIF4F complex formation (3-5).

In human and experimental cancers, free eIF4E levels are commonly elevated via increased $\mathrm{PI} 3 \mathrm{~K} / \mathrm{AKT} / \mathrm{mTOR}$ signaling or eIF4E overexpression $(1,5,6)$. Increased eIF4E expression has been associated with tumor formation and progression in human malignancies including leukemias, lymphomas, and cancers

Nonstandard abbreviations used: ASO, antisense oligonucleotide; 4E-BP, eIF4E binding protein; eIF4E, eukaryotic translation initiation factor 4E; MOE, 2-methoxyethyl; 5'UTR, 5' untranslated region.

Conflict of interest: The authors of this manuscript are employees of Eli Lilly and Company, Isis Pharmaceuticals, and the Wood Hudson Cancer Research Laboratory, a nonprofit research organization supported in part by a grant from Eli Lilly and Company.

Citation for this article: J. Clin. Invest. 117:2638-2648 (2007). doi:10.1172/JCI32044. of the breast, colon, bladder, lung, prostate, and head and neck (reviewed in ref. 1).

In tumors, elevated eIF4E function preferentially and disproportionately enhances translation of select mRNAs. Increased eIF4E function can selectively enable the nucleocytoplasmic transport of potent growth regulatory proteins such as cyclin D1 (7-11). Increased eIF4E function also enhances ribosome loading of mRNAs with lengthy $\mathrm{G}+\mathrm{C}$-rich 5'UTRs, many of which encode potent growth and survival factors involved in malignancy (e.g., c-myc, ODC, VEGF, survivin). Interestingly, most mRNAs, which are characterized by short, unstructured $5^{\prime}$ UTRs (e.g., $\beta$-actin) are largely unaffected by changes in eIF4E activity (1). Consequently, changes in eIF4E most profoundly influence the protein expression of potent regulators of cell growth and survival, i.e., proteins involved in malignancy $(1,2,12)$.

In experimental models, eIF4E overexpression induces cellular transformation, tumorigenesis, invasion, and metastasis (13-15) by selectively upregulating the translation of growth-promoting genes such as ODC (16), cyclin D1 (7-11), and c-myc (13). Furthermore, eIF4E can promote the establishment of autocrine stimulatory loops $(15,17)$. In transgenic models, eIF4E overexpression yields a high incidence of cancer, notably lymphomas, lung adenocarcinomas, hepatomas, and angiosarcomas (18). In the E $\mu$-myc transgenic B cell lymphoma model, wherein myc oncogene expression is driven by the immunoglobulin heavy chain enhancer, eIF4E overexpression accelerates lymphomagenesis $(18,19)$ and imparts 
Human elF4E (M15353) ASO sequence and position

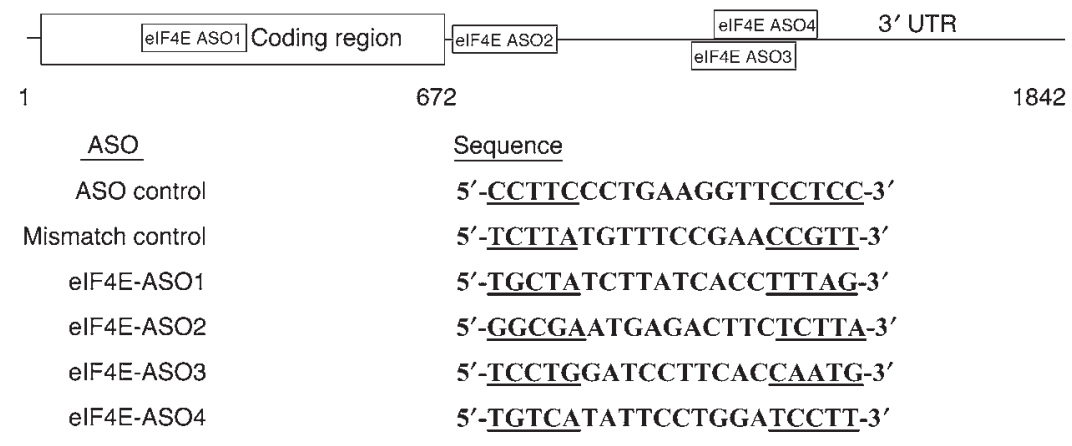

Figure 1

The elF4E ASOs. Four elF4E ASOs were selected for the reduction of elF4E in cultured human and murine cells. The relative position within the human elF4E cDNA (GenBank accession no. M15353) for 4 elF4E-specific ASOs is depicted. The sequences for the universal non-silencing ASO control and the ASO mismatch control are included. The MOE-modified bases are underlined.

resistance to doxorubicin (19). Whereas enhanced eIF4E function promotes malignancy, reducing or inhibiting eIF4E function suppresses malignancy, in concert with reduced expression of potent growth and angiogenesis factors $(6,20-25)$.

Collectively these data indicate that eIF4E may be a promising therapeutic target for the treatment of human malignancies. Further, whereas eIF4E is largely bound to 4E-BPs and inactive in normal tissues, eIF4E function is frequently elevated in cancers, suggesting that cancers may be preferentially susceptible to eIF4Etargeted therapy. Herein we report on eIF4E antisense oligonucleotides (ASOs) designed specifically to target the eIF4E RNA for destruction and to have the plasma and tissue stability necessary for systemic i.v. delivery. Dosed i.v., eIF4E ASOs show single-agent activity in human cancer xenografts, reducing eIF4E expression in xenograft tumor and normal mouse tissues and suppressing tumor growth and angiogenesis without eliciting toxicity. Collectively these data show that tumors are preferentially susceptible to eIF4E-targeted therapy and have prompted the initiation of eIF4E ASO clinical trials for the treatment of human cancers.

\section{Results}

eIF4E-specific ASOs. Currently there are no direct, small molecule eIF4E inhibitors advanced to the clinic, and the development of drug-like small molecules that directly interfere with eIF4E binding to the mRNA cap has proven difficult. We therefore sought a different strategy to target eIF4E for cancer therapy. We chose to develop an eIF4E-specific second-generation ASO that could be administered systemically for the treatment of human cancers. ASOs recognize and hybridize to target mRNA by Watson-Crick base pairing and trigger RNase $\mathrm{H}$-mediated RNA destruction. Earlier generation ASOs lacked the nuclease resistance and tissue stability necessary for systemic therapy. By contrast, the second generation ASOs have been reengineered, retaining the phosphorothioate backbone core (to promote $\mathrm{RNase} \mathrm{H}$-mediated degradation) but are now flanked with five 2-methoxyethyl-modified (MOE-modified) bases (underlined in Figure 1) to improve potency, nuclease resistance, and tissue half-life (26-29).

Overlapping MOE-gapmer ASOs, targeting the entire eIF4E mRNA sequence (Figure 1), were evaluated for the ability to reduce eIF4E RNA expression. The eIF4E sequence is well conserved across species. We therefore chose ASOs that would target both human and murine eIF4E so that we could simultaneously assess the in vivo effects of eIF4E reduction on human xenograft tissues and normal mouse tissues. These eIF4E ASOs reduce eIF4E RNA expression by more than $50 \%$ at concentrations less than $25 \mathrm{nM}$ in human tumor cells and murine endothelial cells, as measured by quantitative RT-PCR (Figure 2A).

eIF4E ASOs reduce expression of eIF4E and eIF4E-regulated proteins. We next assessed ASOmediated reduction of eIF4E protein in cultured human tumor and endothelial cells by Western blotting with cells harvested 24, 48, or 72 hours after transfection. These analyses defined 72 hours after transfection as being the optimal time frame for eIF4E protein reduction (data not shown) in a wide range of human tumor cells, including head and neck cancers (FaDu, SW579), prostate cancers (PC-3, CWR-22Rv1), breast cancers (MDA-MB-231) and non-small cell lung cancers (NCI-H460) (Figure 2, B-D). eIF4E ASOs also reduced eIF4E expression in human umbilical vein endothelial cells (HUVECs) (Figure 2C).

Many key proteins involved in malignancy are translationally controlled, including the potent angiogenic factors VEGF and FGF-2, the oncogenes cyclin D1 and c-myc, the antiapoptotic proteins of the $\mathrm{Bcl}$ family, as well as the inhibitor of apoptosis protein survivin (refs. 30, 31; see ref. 1 for a more extensive list of translationally controlled proteins involved in malignancy). Indeed, modulation of eIF4E can directly affect the expression of many of these malignancyrelated proteins (reviewed in refs. 1, 30). We therefore evaluated whether ASO-mediated eIF4E reduction would affect the expression of these malignancy-related proteins. We specifically chose to evaluate the oncogenes c-myc and cyclin D1, the angiogenesis factor VEGF, and the antiapoptotic proteins Bcl-2 and survivin. The expression of cyclin D1, c-myc, VEGF, Bcl-2, and survivin was substantially decreased along with reduced eIF4E expression 72 hours after transfection with 4E-ASO4 (Figure 2D). By contrast, $\beta$-actin expression was largely unaffected by reduced eIF4E expression (Figure 2), consistent with the notion that altering eIF4E levels selectively affects expression of growth regulatory proteins (1).

eIF4E ASOs do not substantially affect global protein synthesis. Though translation of many growth and survival factors is heavily dependent upon the availability of eIF4E, most cellular mRNAs require only minimal eIF4E function to be efficiently translated $(1,2)$. Consequently, modulating eIF4E function has limited effects on global protein synthesis $(1,20)$. We therefore determined the extent to which eIF4E reduction by eIF4E ASOs would affect global protein synthesis rates. MDA-MB-231 cells (a highly invasive human breast cancer cell line) were transfected with 4E-ASO 4 or $4 \mathrm{E}-\mathrm{ASO} 2 .{ }^{35} \mathrm{~S}$ incorporation into total protein was measured in parallel with eIF4E RNA expression 72 hours after transfection. Though eIF4E RNA expression was reduced by approximately $80 \%$ in MDA-MB-231 cells transfected with 75 nM ASO, there was only a marginal change in the ${ }^{35} \mathrm{~S}$-labeled protein expression pattern (Figure 3, A and B). When normalized to total RNA, ${ }^{35} \mathrm{~S}$ incorporation into newly synthesized protein was reduced $20 \%$ when compared with the mismatch ASO or mock-transfected controls (Figure 3C). Similarly, ${ }^{35} \mathrm{~S}$ incorporation into newly synthesized 

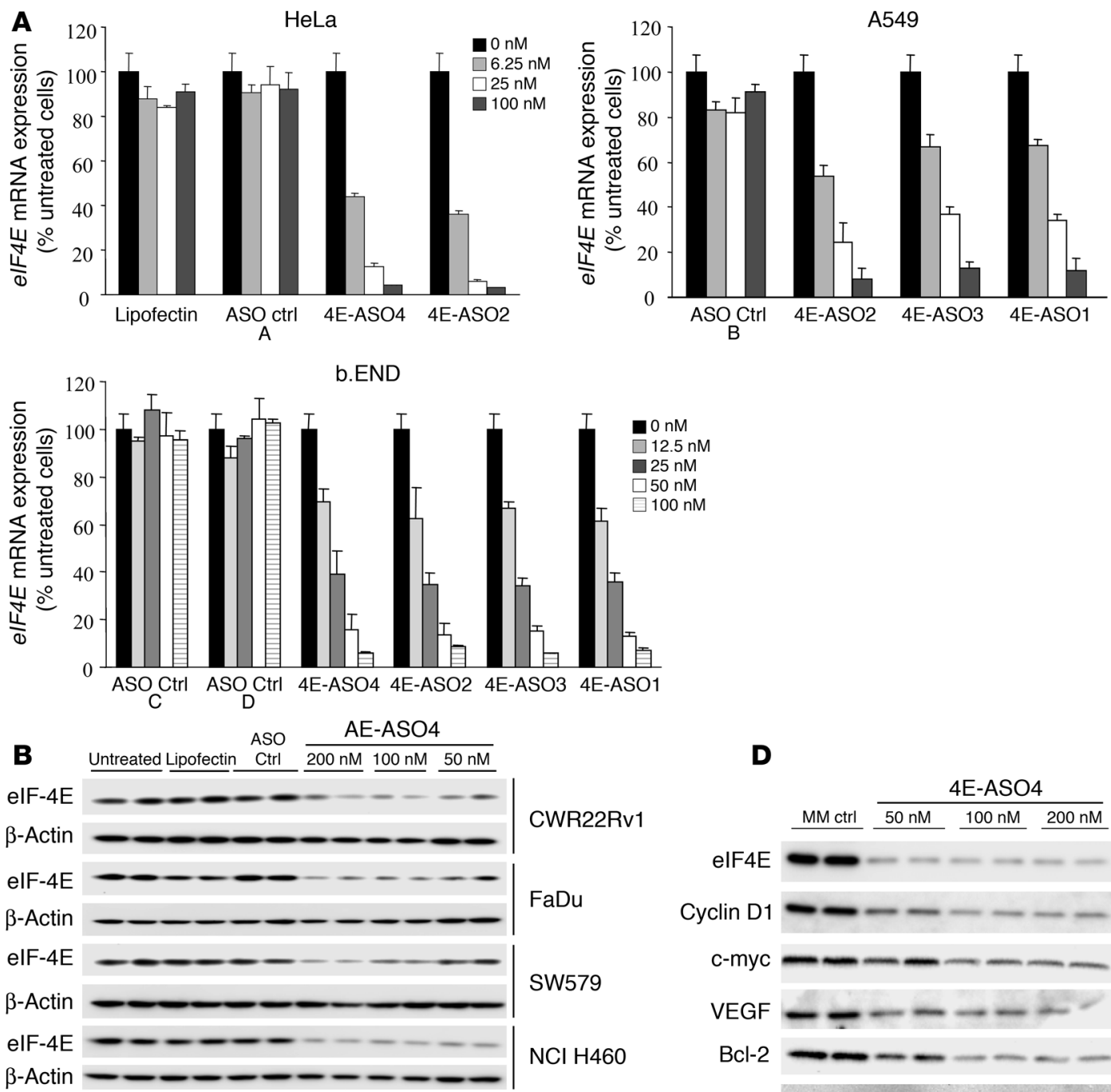

CWR22Rv1

D
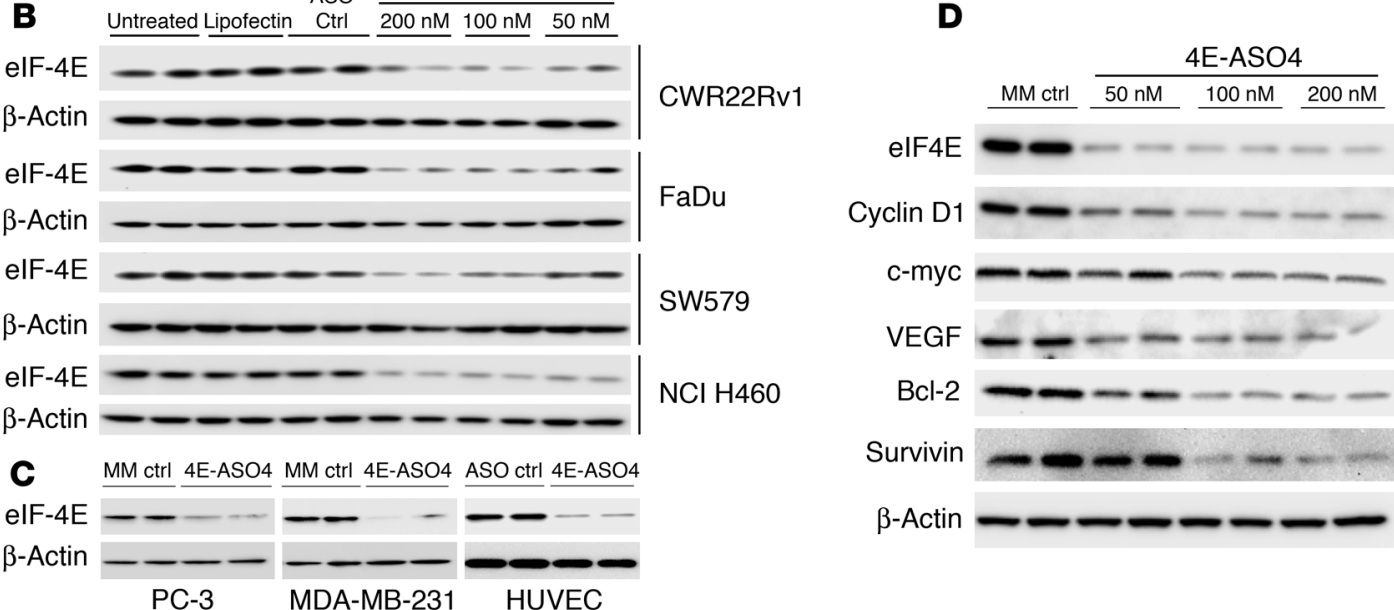

Figure 2

elF4E expression is reduced in cultured human and murine cells. (A) elF4E expression was assessed by quantitative RT-PCR. Representative data are shown for human cervical carcinoma cells (HeLa), human non-small cell lung cancer cells (A549), and murine endothelial cells (b.END cells) 24 hours after transfection. Cells were transfected using lipofectin, the elF4E-specific ASOs, or the non-silencing ASO controls at the indicated concentrations (5'-3', ASO ctrIA, GGATAGAACGCGAAAGCTTG; ASO ctrIB, GTACAGTTATGCGCGGTAGA; ASO ctrIC, CGTTATTAACCTCCGTTGAA; ASO ctrID, TTAGAATACGTCGCGTTATG). Data are presented as the percentage of elF4E in control untransfected cells. (B-D) elF4E protein expression was evaluated by Western blotting from cell lysates harvested 72 hours after transfection. Each blot was reprobed for $\beta$-actin to control for loading and transfer variations. Representative data are shown for human prostate cancer cell lines (CWR22Rv1 and PC-3), head and neck cancer cell lines (FaDu and SW579), a human breast cancer cell line (MDA-MB-231), a human non-small cell lung cancer cell line (NCl-H460), and the HUVEC. (B) Cells were transfected with 50, 100, or $200 \mathrm{nM} \mathrm{4E-ASO4}$ or the ASO control. (C) Data shown represent cells transfected with $200 \mathrm{nM} 4 \mathrm{E}-\mathrm{ASO} 4$ or the mismatch (MM) control. (D and E) MDA-MB-231 breast cancer cells were transfected with 4E-ASO4 or mismatch control ASO. Protein was harvested 72 hours after transfection, and lysates were analyzed by Western blotting for the indicated proteins.

protein was also only marginally reduced in HeLa cells transfected with $100 \mathrm{nM} 4 \mathrm{E}-\mathrm{ASO} 4$ or 4E-ASO2, though eIF4E RNA levels were decreased more than $80 \%$ (data not shown). By contrast, cycloheximide, which completely blocks global protein synthesis, nearly completely reduced ${ }^{35} \mathrm{~S}$ incorporation (Figure 3 ). These data demonstrate that eIF4E reduction by 2 different ASOs has only a lim- ited effect on total protein synthesis, an effect both qualitatively and quantitatively distinct from that of cycloheximide.

eIF4E ASO transfection induces apoptosis. eIF4E has been implicated in the regulation of cellular growth and survival $(1,30-35)$. Blocking the interaction of eIF4E with eIF4G using specific peptides can induce apoptosis (32). Moreover, eIF4E overexpression can 
A

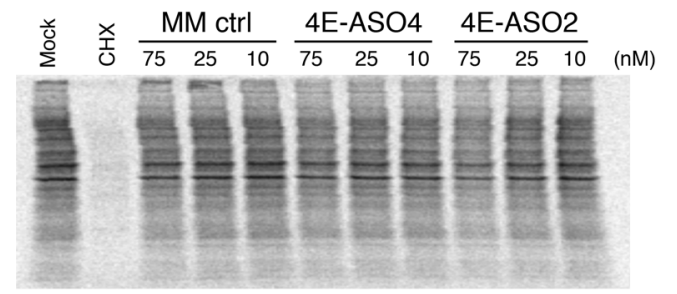

B
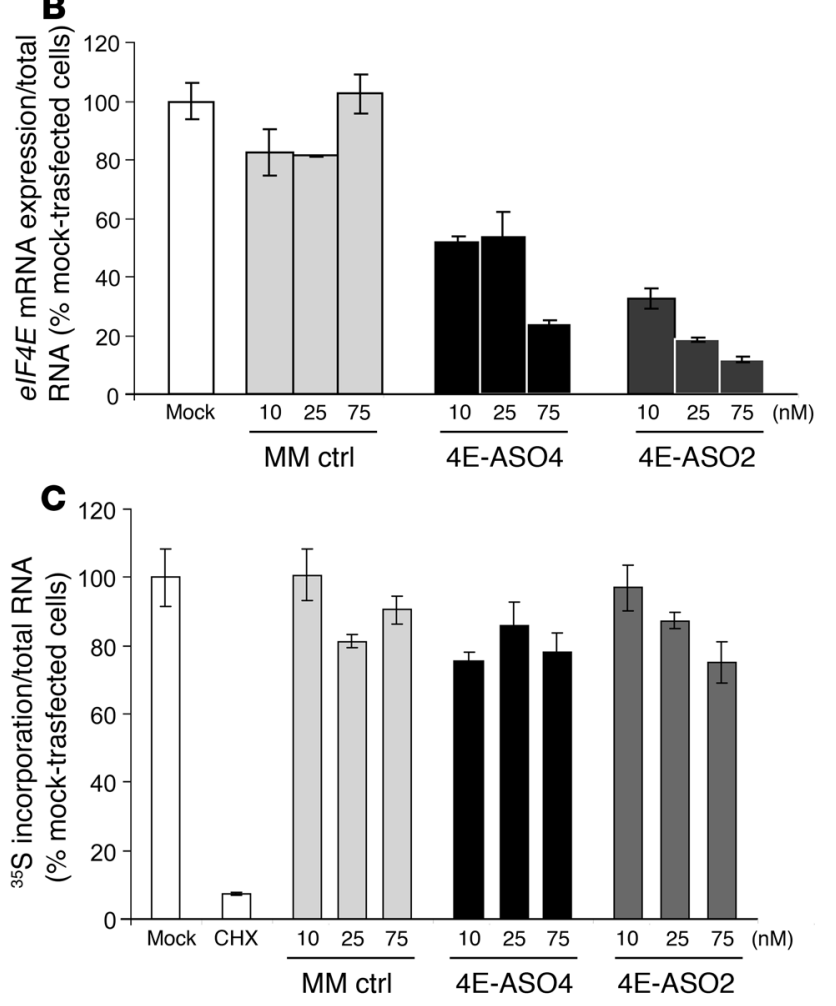

protect cells from apoptosis $(31,33-35)$, in part by regulating the translation of many mRNAs encoding proteins involved in regulating apoptosis such as survivin $(30,31)$. We therefore sought to determine whether ASO-mediated eIF4E reduction might induce apoptosis. Transfection of MDA-MB-231 breast cancer cells with 4E-ASO4 induced substantial apoptosis as measured by TUNEL staining (Figure 4A). Nearly 40\% of MDA-MB-231 cells were positive for TUNEL staining and 50\% were positive for activated caspase- 3 staining 72 hours after transfection with $100 \mathrm{nM} 4 \mathrm{E}-\mathrm{ASO} 4$, which typically reduces eIF4E levels by approximately $80 \%$ (Figure 2D). By contrast, in the MDA-MB-231 cells transfected with the mismatch control ASO, 10\% of cells were TUNEL- and activated caspase-3-positive (Figure 4B). Accordingly, there was a marked reduction in cell number, which was evident by the reduced number of Hoechst-stained nuclei (Figure 4A). Likewise, the percentage of TUNEL- and activated caspase-3-positive cells was also increased in the non-small cell lung cancer line $\mathrm{H} 460$ after transfection with $100 \mathrm{nM}$ 4E-ASO4 when compared with cells transfected with the mismatch ASO control (Figure 4B). These data demonstrate that 4E-ASO-mediated reduction in eIF4E induces apoptosis.

eIF4E ASO transfection blocks endothelial cell tube formation in vitro. The AKT/mTOR signaling pathway, which liberates eIF4E from the 4E-BPs, is activated in endothelial cells by angiogenic factors (36). It is therefore plausible that eIF4E plays a direct role in the

\section{Figure 3}

Reduction of elF4E and global protein synthesis. (A) The human breast cancer cell line MDA-MB-231 was transfected for 72 hours with $4 \mathrm{E}-\mathrm{ASO} 4,4 \mathrm{E}-\mathrm{ASO} 2$, or the mismatch ASO control at the indicated concentrations. Cells were labeled 72 hours after transfection with ${ }^{35}$ S-methionine/cysteine (Promix). As a positive control for blocking total protein synthesis, cells were pretreated with cycloheximide (CHX) 4 hours before the addition of Promix. Equal protein was loaded per lane on an SDS-PAGE gel, electrophoresed, dried, and exposed to a phosphorimager screen overnight. (B) RT-PCR analyses for elF4E expression normalized to total RNA as determined by Ribogreen stain are depicted for MDA-MB-231. (C) ${ }^{35} \mathrm{~S}$ incorporation per lane was normalized to total RNA from the same sample. Data represent 2 separate experiments in MDA-MB-231.

endothelial cell response to angiogenic stimuli, though this has never been explored. We therefore evaluated whether eIF4E reduction might suppress the response of endothelial cells to angiogenic stimuli. Specifically, we chose to evaluate the formation of tube or chord-like structures by HUVECs. Though this large endothelial cell model may not fully reflect the process of angiogenesis, these cells nevertheless provide a useful model system for evaluating the response of endothelial cells to angiogenic stimuli. The formation of these vessel-like structures by HUVECs cultured on Matrigel was scored semi-quantitatively on a scale of 1 to 5 after eIF4E ASO or control ASO transfection (37). Two separate ASO controls showed only marginal effects on tube formation, whereas 4E-ASO4 yielded a dose-dependent decrease in tube formation, corresponding to a dose-dependent reduction in eIF4E RNA expression of greater than $80 \%$ (Figure 4, C and D). 4E-ASO2 also suppressed endothelial tube formation, though to a lesser extent than 4E-ASO4 (Figure 4C).

We next confirmed these analyses in a separate HUVEC cell system. After transfection with the 4E-ASO4, HUVECs were replated on a bed of dermal fibroblasts (38) and incubated 6 days to allow for the formation of chordlike structures. 4E-ASO4 transfection reduced eIF4E protein expression by approximately 65\% (after normalization to $\beta$-actin) 48 hours after transfection and markedly reduced the formation of the elongated endothelial chordlike structures by HUVECs when compared with those treated with the mismatch control ASOs (Figure 4E). Collectively, the results of these assays suggest that eIF4E reduction may influence the response of endothelial cells to angiogenic stimuli.

Systemic eIF4E ASO dosing reduces eIF4E expression in xenograft tumors, suppressing tumor growth and angiogenesis. MOE-gapmer ASOs such as the eIF4E ASOs can be administered systemically (i.e., intravenously) (26-29). Importantly, in recent human clinical trials, second-generation MOE-gapmer ASOs administered i.v. showed dose-dependent ASO accumulation and target reduction in primary prostate cancer tissues (39). These data clearly show that second-generation ASOs can accumulate in human cancer tissues, successfully reducing target mRNA and protein. We therefore sought to determine whether i.v. administration of 4E-ASO4 would reduce eIF4E expression in human cancer xenograft tissue and whether this might affect xenograft tumor growth.

A role for eIF4E in the genesis and maintenance of breast cancers has been established (6). We therefore chose to evaluate the impact of i.v. eIF4E ASO administration on eIF4E expression and tumor growth in the MDA-MB-231 breast cancer xenografts. 4E-ASO4 was administered at $50 \mathrm{mg} / \mathrm{kg}$ thrice weekly by i.v. bolus injection to nude mice bearing MDA-MB-231 xenografts. Treatment con- 

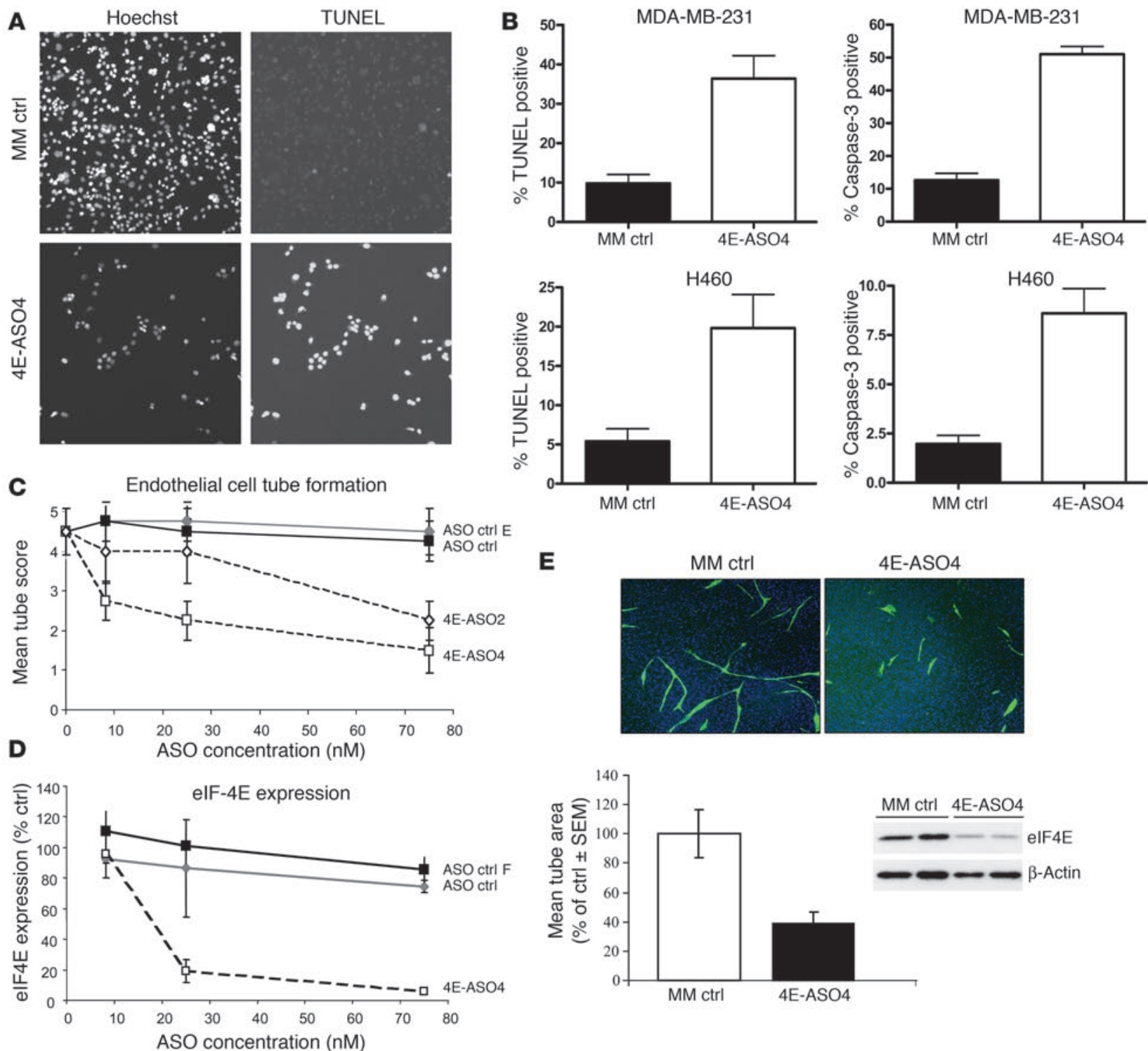

Figure 4

eIF4E ASO transfection induces apoptosis and suppresses endothelial cell tube formation. (A) MDA-MB-231 human breast cancer cells were transfected for 72 hours with $100 \mathrm{nM} 4 \mathrm{E}-\mathrm{ASO} 4$ or mismatch control. Representative photomicrographs illustrate the dramatic increase in TUNEL staining after 4E-ASO4 transfection. Nuclear staining is revealed by Hoechst dye. (B) The mean percentage of cells positive for TUNEL or activated caspase-3 is depicted for both MDA-MB-231 and $\mathrm{H} 460$ non-small cell lung cancer cells $( \pm$ SEM). Data in A and B are representative of more than 4 separate determinations. (C) HUVECs were plated on Matrigel and transfected with the 4E-ASO4, 4E-ASO2, or non-silencing ASO controls as indicated (ASO ctrlE, 5'-TGTTACAGTCTTGTACCCTT-3' and "randomer" ASO ctrlF, a random mix of 420 possible 20-mer nucleotide sequences). The formation of vessel-like tubes was scored semi-quantitatively on a scale of 1 to 5 . Data are presented as the mean tube score from 4 separate determinations \pm SD. (D) Mean elF4E expression \pm SEM was evaluated from parallel plates by quantitative RT-PCR for the controls and the 4E-ASO4-transfected HUVECs. RT-PCR for the cells treated with 4E-ASO2 was not performed. (E) HUVECs were transfected for 48 hours with $150 \mathrm{nM}$ 4E-ASO4 or mismatch control ASO, replated on dermal fibroblasts, and incubated for 6 days to assess the formation of chord-like structures. Endothelial cells were visualized immunohistochemically with an anti-CD31 antibody (green). Hoechst stain reveals the nuclei of the dermal fibroblasts (blue). Western blot analyses of elF4E expression 48 hours posttransfection are shown. Data are representative of 5 separate experiments.

tinued for more than 2 months and dramatically suppressed the growth of these tumors (Figure 5A; $P<0.01$ versus mismatch control-treated tumors). To increase our throughput for the analysis of eIF4E protein levels in xenograft tissues, we developed and validated an eIF4E ELISA. MDA-MB-231 xenograft tumor tissues harvested at the end of the study showed a significant $64 \%$ reduction in eIF4E expression as measured by ELISA $(P=0.011$; Figure 5A, right). Importantly, the body weight of these mice was unaffected by this treatment (data not shown).

We sought next to determine whether additional xenograft models might also be affected by eIF4E ASO administration. Prostate cancers frequently show enhanced $\mathrm{AKT} / \mathrm{mTOR}$ pathway signaling 

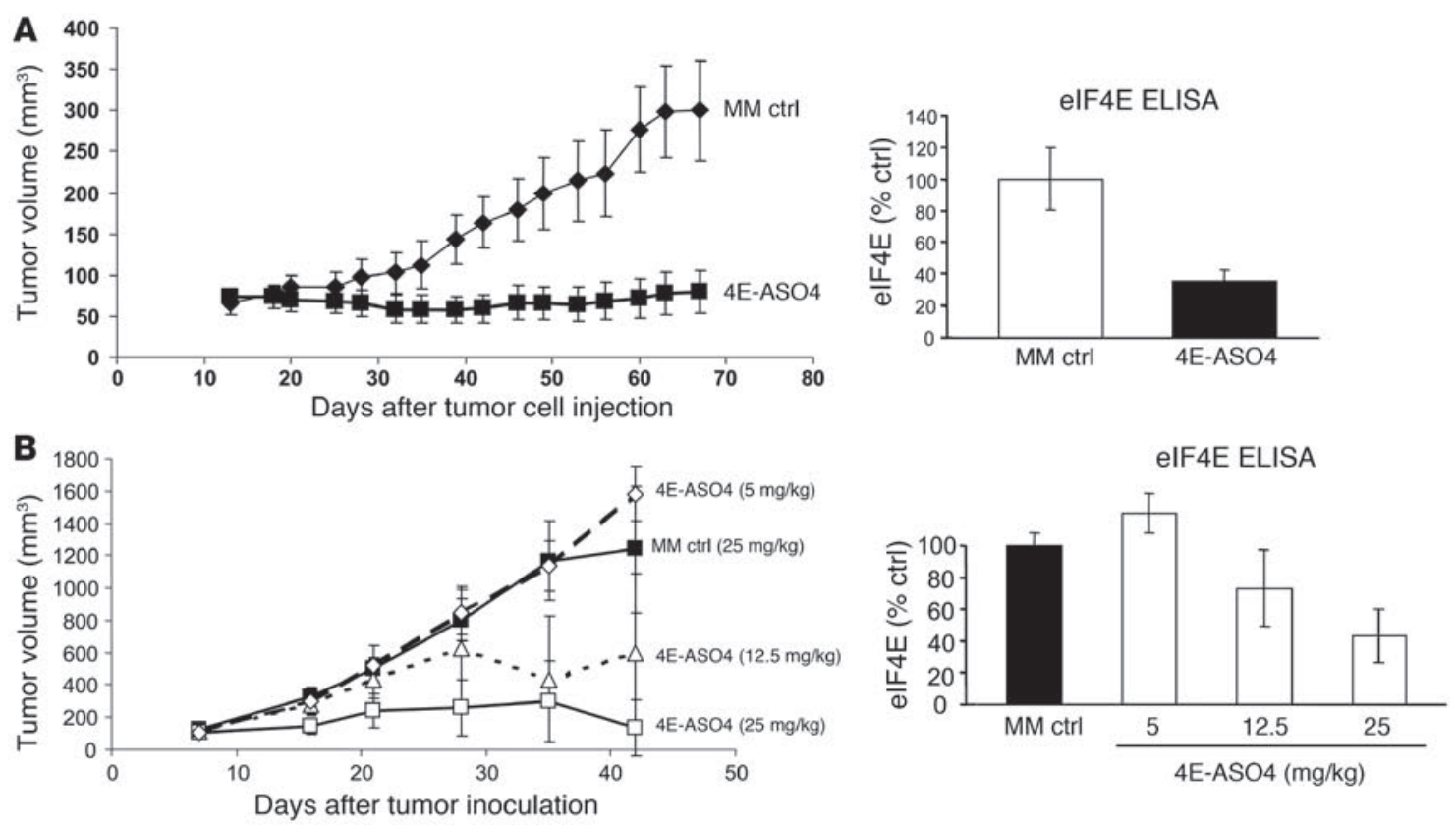

C
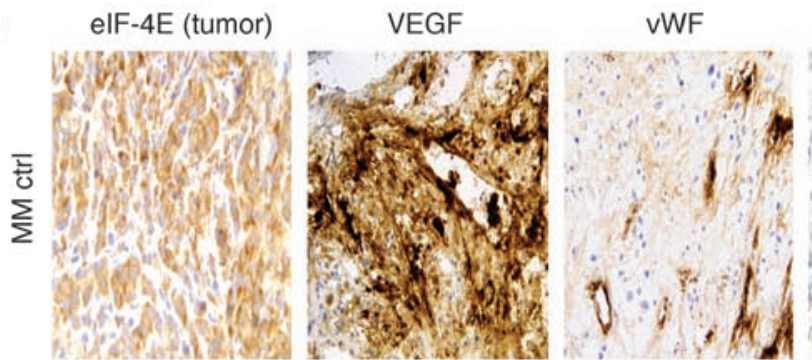

TUNEL

Ki-67

elF-4E (liver)
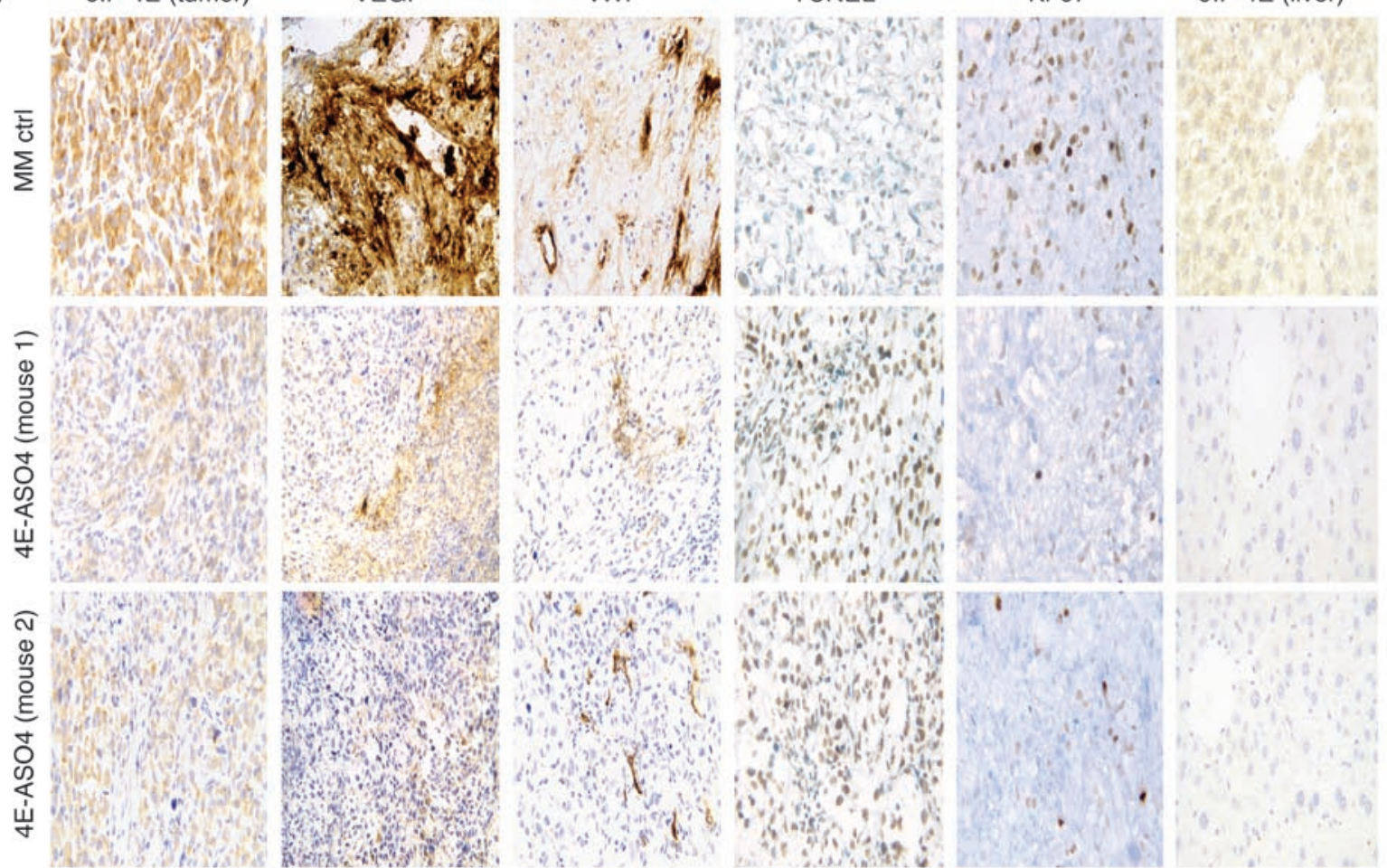

\section{Figure 5}

Administration of 4E-ASO4 i.v. suppresses elF4E expression and xenograft tumor growth. (A) Nude mice bearing MDA-MB-231 human breast cancer xenografts $\left(\sim 100 \mathrm{~mm}^{3}\right)$ were dosed i.v. with 4E-ASO4 or mismatch control at $50 \mathrm{mg} / \mathrm{kg}(n=10 / \mathrm{group})$ thrice weekly after an initial $100 \mathrm{mg} /$ $\mathrm{kg}$ loading dose. elF4E expression was evaluated by ELISA from xenograft tissues (right). (B) Nude mice bearing PC-3 human prostate cancer xenografts $\left(-100 \mathrm{~mm}^{3}\right)$ were dosed i.v. with 4E-ASO4 or mismatch control ( $\left.n=8 / \mathrm{group}\right)$ after an initial loading dose of $50 \mathrm{mg} / \mathrm{kg} \mathrm{ASO}$, followed by the indicated doses thrice weekly. elF4E expression was evaluated by ELISA from xenograft tissues (right). For A and B, 4E-ASO4 administration significantly suppressed xenograft tumor growth versus mismatch ASO controls (repeated measures ANOVA with covariates of log baseline tumor size and log baseline body weight, $P=0.01$ for MDA-MB-231; $P=0.015$ for PC-3). elF4E protein expression was reduced significantly by $4 \mathrm{E}-\mathrm{ASO} 4$ administration (64\% reduction, $P=0.011$ for MDA-MB-231; $56 \%$ reduction, $P=0.012$ for $P C-3$ ). Data are plotted as mean tumor volume versus time after tumor inoculation \pm SEM and are representative of 3 separate tumor xenograft studies with PC-3 and 2 separate xenograft studies with MDA-MB-231. (C) PC-3 prostate carcinoma xenograft tissue was evaluated by immunohistochemistry for elF4E, VEGF, vWF, TUNEL, and Ki-67. elF4E staining in liver from these same mice is also shown. Representative data are shown for mismatch control-treated tumors and tumors from 2 separate mice treated with $25 \mathrm{mg} / \mathrm{kg}$ 4E-ASO4. The most intensely stained sections from each tumor are shown. 
as well as increased eIF4E expression, particularly in advanced disease (refs. 40, 41, J.R. Graff and J.H. Carter, unpublished observations), suggesting a role for eIF4E in human prostate cancers. Additionally, second-generation ASOs accumulate and reduce target gene expression in human prostate cancer tissue (39). We therefore chose to evaluate the impact of 4E-ASO4 administration on the growth of PC-3 human prostate cancer xenografts. PC-3 xenograft tumor growth was significantly reduced in mice dosed with $25 \mathrm{mg} /$ $\mathrm{kg}$ 4E-ASO4 when compared with mice treated with the $25 \mathrm{mg} / \mathrm{kg}$ mismatch control ASO $(P=0.015$; Figure $5 \mathrm{~B})$. The group dosed with $12.5 \mathrm{mg} / \mathrm{kg}$ 4E-ASO4 showed an intermediate effect, failing to show statistical significance when compared with either the mismatch control group or $25 \mathrm{mg} / \mathrm{kg}$ 4E-ASO 4 group (Figure $5 \mathrm{~B}$ ). The group mean tumor volume was unaffected by dosing with $5 \mathrm{mg} / \mathrm{kg}$ 4E-ASO4. Importantly, there was no change in mean body weight related to eIF4E ASO treatment (data not shown).

Tumors were harvested at the end of this study to evaluate eIF4E expression. The eIF4E ELISA data showed a dose-related reduction in eIF4E protein expression, paralleling the anti-tumor effects of the eIF4E ASO (Figure 5B, right). The $25 \mathrm{mg} / \mathrm{kg} \mathrm{4E-}$ ASO 4 group showed a $56 \%$ reduction in eIF4E protein expression relative to the mismatch control (normalized to the GAPDH ELISA data, $P=0.012$; normalized to total protein concentration, $P=0.017)$. The $5 \mathrm{mg} / \mathrm{kg}$ dose group showed no change in eIF4E levels ( $P=0.477$ versus mismatch control; Figure $5 \mathrm{~B}$, right), consistent with having no effect on tumor growth. The $12.5 \mathrm{mg} / \mathrm{kg}$ dose group, which showed an intermediate effect on tumor growth, showed a slight but statistically insignificant reduction in eIF4E expression compared with the mismatch control $(P=0.323$ versus mismatch control; Figure 5B, right). To complement and confirm these ELISA data, we also examined eIF4E protein expression by immunohistochemistry. In mice treated with $25 \mathrm{mg} / \mathrm{kg}$ 4E-ASO4, eIF4E immunostaining in xenograft tumors was markedly reduced when compared with tumors from mice treated with the mismatch control (Figure 5C).

The eIF4E ASOs suppressed VEGF expression in cultured tumor cells (Figure 2D) and inhibited tube formation by cultured endothelial cells (Figure 4, C-E), suggesting that the reduction of eIF4E may have antiangiogenic effects. We therefore examined the PC-3 xenograft tumors for VEGF expression and endothelial content. Immunostaining showed that VEGF protein expression was reduced in PC-3 tumor xenografts treated with $25 \mathrm{mg} / \mathrm{kg}$ 4E-ASO4 compared with those treated with the mismatch control ASO (Figure 5C). Immunostaining for $\mathrm{vWF}$, an endothelial-specific marker (42), was also reduced in both intensity and area in the tumors treated with the 4E-ASO4 when compared with mismatch control tumors (Figure 5C). These data are suggestive of an antiangiogenic effect of the eIF4E ASO in treated xenograft tumors.

In cultured human cancer cells, the 4E-ASO4 robustly induced apoptosis. We therefore sought to determine whether apoptosis might be evident in the tumors treated with 4E-ASO4. In tumors from mice dosed with $25 \mathrm{mg} / \mathrm{kg}$ 4E-ASO4, the number of TUNELpositive cells was markedly increased for each $\times 40$ field in the 4EASO4-treated tumors $(88.4 \pm 20.9$ cells/field) compared with the mismatch-treated tumors $(10.1 \pm 1.3$ cells/field; $P=0.020$, 2-tailed Student's $t$ test) (Figure 5C). We also evaluated proliferation in these xenograft tumors by Ki-67 immunostaining, a marker for mitotic cells. Tumors from mice treated with 4E-ASO4 showed a marked reduction in Ki-67 positivity (Figure 5C). In tumors from mice treated with the mismatch control, there were $83.2 \pm 5.0 \mathrm{Ki}-67$-positive cells per $\times 40$ field, whereas in tumors from mice treated with $4 \mathrm{E}-\mathrm{ASO} 4$, the Ki-67-positive cells were dramatically reduced to $21.1 \pm 4.8$ cells per field ( $P<0.001,2$-tailed Student's $t$ test). These data indicate that systemic administration of the 4E-ASO4 elicits apoptosis and reduces proliferation in xenograft tumors.

eIF4E expression is reduced in normal mouse tissues without toxicity. The mRNA cap-binding protein eIF4E is necessary for cap-dependent translation, which accounts for the majority of cellular translation (1). As such, eIF4E is considered an essential part of the protein synthesis machinery. To assess whether the reduction of eIF4E might deleteriously affect normal tissues, we expressly engineered and selected ASOs that could effectively target both human and murine eIF4E (see Figure 2). There was neither a change in body weight of the xenograft-bearing mice treated with the eIF4E ASO nor signs of illness or distress. We therefore examined eIF4E protein levels in the livers of these tumor-bearing mice, particularly because the liver is a tissue where ASOs preferentially accumulate (26-29). eIF4E protein was reduced in these livers by eIF4E ASO treatment when compared with livers from mice treated with the mismatch control ASOs (Figure 5C) (eIF4E was reduced $>80 \%$ in these tissues by ELISA; data not shown).

To evaluate more fully the impact of eIF4E reduction on normal function, we dosed non-tumor bearing mice twice weekly at 40 $\mathrm{mg} / \mathrm{kg}(80 \mathrm{mg} / \mathrm{kg} /$ wk compared with $75 \mathrm{mg} / \mathrm{kg} /$ wk in the xenograft studies) for 3 weeks with each of the 4 eIF4E ASOs. We measured eIF4E expression in liver as well as liver weight, body weight, spleen weight, and plasma levels of liver transaminases to detect liver injury. eIF4E RNA expression was reduced by up to $80 \%$ by these eIF4E ASOs (Figure 6A) (Western blotting showed a similar eIF4E reduction for each 4E-ASO; data not shown). Despite this profound reduction in eIF4E expression, there was no appreciable change in liver weight, spleen weight, body weight, or liver transaminase levels in these mice when compared with saline treatment or treatment with a non-silencing ASO control (Figure 6, B-D). Collectively, these data indicate that eIF4E expression levels can be profoundly reduced in normal tissues without eliciting signs of illness or distress, changing body or organ weight, or affecting plasma levels of liver transaminases.

\section{Discussion}

Elevated eIF4E function has been repeatedly implicated in malignancy, enhancing the translation of key malignancy-related proteins, promoting angiogenesis, enabling tumor growth and progression, and imparting chemoresistance $(1,2,6,18,19)$. eIF4E is therefore a rational target for novel anticancer therapeutics. With this report, we now show that eIF4E can be selectively targeted in human cancer xenograft tissues for destruction by second-generation ASOs. Specifically, we show that eIF4E ASOs decreased eIF4E RNA and protein expression in cultured human cancer and endothelial cells as well as murine cell lines. Moreover, eIF4E reduction in human tumor cells robustly induced apoptosis and suppressed expression of VEGF, cyclin D1, c-myc, Bcl-2, and survivin. eIF4E reduction also inhibited the formation of vessel-like structures by cultured human endothelial cells. Furthermore, systemic administration of eIF4E ASOs suppressed eIF4E expression in normal mouse tissues and in xenografted human cancer tissues, repressing xenograft tumor growth without eliciting toxicity. Indeed, in non-tumor bearing mice, eIF4E-specific ASOs dramatically reduced eIF4E expression in the mouse liver without appreciably altering liver weight, spleen weight, body weight, or 

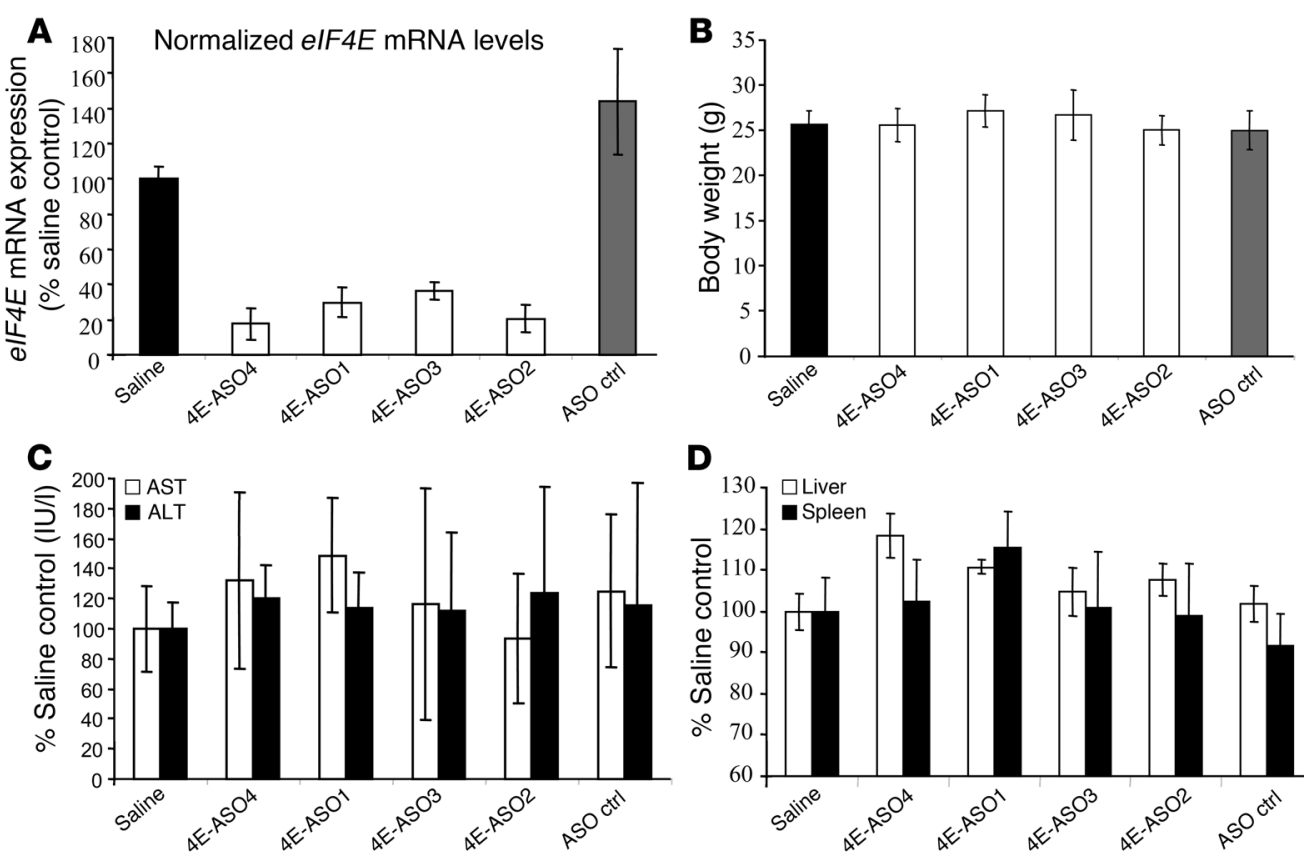

D

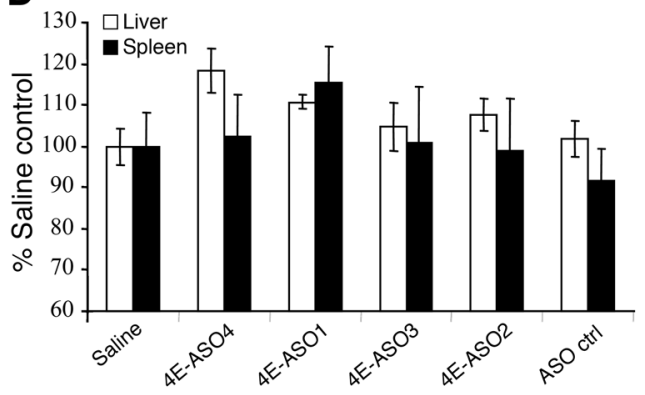

\section{Figure 6}

ASO-mediated reduction of elF4E is well tolerated. Four distinct elF4E-specific ASOs (4E-ASO1, -2, -3, and -4) and the control ASO were administered i.v. at $40 \mathrm{mg} / \mathrm{kg}$ twice weekly $(80 \mathrm{mg} / \mathrm{kg} / \mathrm{wk}$ ) for 3 weeks. At the end of the 3-week study, mice were weighed and liver, spleen, and blood were harvested. (A) eIF4E expression was evaluated by quantitative RT-PCR in liver, a preferential site of ASO accumulation. (B and D) Body weights, liver weights, and spleen weights were recorded. (C) Plasma was analyzed for liver transaminase (aspartate aminotransferase [AST] and alanine aminotransferase $[A L T]$ ) levels. plasma levels of liver transaminases. These preclinical data have provided the foundation for further evaluation of eIF4E ASOs in human clinical trials for the treatment of cancer.

Our data suggest that targeting eIF4E may suppress tumor growth through multiple mechanisms. eIF4E ASOs can affect tumor cells directly. eIF4E ASO transfection reduced eIF4E expression and diminished the expression of the oncogenes, c-myc and cyclin D1, and the anti-apoptotic proteins survivin and $\mathrm{Bcl}-2$. Accordingly, the eIF4E ASO robustly induced apoptosis and reduced cell viability in cultured tumor cells (Figure 4). Increased apoptosis and reduced cellular proliferation were also evident in xenograft tumor tissues from mice treated with 4E-ASO4 (Figure 5C). Consistent with these effects, 4E-ASO 4 profoundly suppressed the growth of human breast and prostate xenograft tumors (Figure 5, A and B).

eIF4E ASO treatment may also suppress tumor growth by suppressing angiogenesis. eIF4E reduction suppressed expression of the potent angiogenic factor VEGF by cultured human tumor cells (Figure 2D) and inhibited the formation of endothelial tube structures by cultured human endothelial cells (Figure 4, C-E). Furthermore, immunostaining for VEGF and for the endothelial cell marker vWF was reduced in conjunction with eIF4E reduction in PC-3 xenograft tumor tissues treated with 4E-ASO4 (Figure 5C). Collectively these data suggest that eIF4E reduction may affect tumor cells directly and may also affect tumor-related angiogenesis.

Our data also now show for what we believe to be the first time that tumor tissues may be more susceptible than normal tissues to the reduction of eIF4E. Systemic delivery of eIF4E ASOs routinely reduced eIF4E expression by approximately $80 \%$ in the mouse liver but did not elicit changes in body weight, liver or spleen weight, or levels of liver transaminases (Figure 6). Similarly, in xenograftbearing mice, eIF4E was routinely reduced by approximately $80 \%$ in the mouse liver (see Figure 5C) without eliciting changes in body weight or any signs of illness or distress. Yet in these same mice tumor growth was significantly suppressed (Figure 5, A and B), coinciding with an approximately 50\% reduction in eIF4E expression within the tumor. These data thereby indicate that reduced eIF4E expression suppresses tumor growth but does not, at least under these conditions, deleteriously affect normal tissues.

The differential susceptibilities of tumor tissue and normal tissue to eIF4E reduction may be related to the following: In normal resting cells, eIF4E would largely be inactive, as it would be complexed with the inhibitory 4E-BPs in the absence of mitogenic signals (3-6). Reducing the expression of an inactive protein may have minimal impact on that cell or tissue. In addition, most cellular mRNAs (i.e., those necessary for normal cell or tissue maintenance, for example $\beta$-actin) require only minimal eIF4E function to be translated (1). On the other hand, the continued expression of potent growth and survival factors, most of which are encoded by mRNAs with lengthy G- and C-rich 5'UTRs, is particularly dependent upon elevated eIF4E function. Hence eIF4E reduction would most profoundly impact the translation of these potent growth and survival factors, which are necessary to sustain tumor growth and survival. Such eIF4E reduction would have a more modest effect on the expression of proteins necessary for normal tissue maintenance (1). Indeed, our data showing that eIF4E reduction has a limited effect on global protein synthesis are consistent with this notion (Figure 3). It is however plausible that the reduction of eIF4E in normal tissues could have an impact on the ability of these tissues to respond to stress, as might occur in response to radiation or standard chemotherapy treatment.

The data presented herein demonstrate for what we believe to be the first time the feasibility of employing eIF4E-specific therapy for the treatment of human malignancies. These data illustrate that systemic administration of second-generation ASOs, which have been expressly engineered for enhanced plasma and tissue stability, can effectively target eIF4E for destruction in xenograft tumor tissues, significantly suppressing tumor growth and affecting angiogenesis. Moreover, even with profound reduction of eIF4E expression in mouse liver ( $80 \%)$, mice showed no signs of 
illness or distress, no changes in body, liver, or spleen weight, and no increases in plasma levels of liver transaminases. These data reinforce the notion that targeting eIF4E will selectively affect tumor tissues compared with normal tissues (1) and provide the foundation for advancing eIF4E ASOs to clinical trials for the treatment of human malignancies.

\section{Methods}

ASO generation and selection. Second-generation ASOs with a phosphorothioate backbone and flanking bases modified with a methoxyethyl group at the 2 ' position of the sugar were screened for the reduction of eIF4E RNA levels in cultured human and mouse cell lines by quantitative realtime RT-PCR. These ASOs did not show significant alignment with the eIF4E homologous proteins eIF4E-2 or eIF4E-3. A variety of nonsilencing control MOE-gapmer ASOs were used throughout the course of this work. The sequences for the universal ASO control, the mismatch ASO control, and 4 eIF4E-specific ASOs are shown in Figure 1.

Cell culture, RT-PCR, Western blotting, and ELISA analyses. Cells were cultured as described in ref. 43. Murine b.END cells and all tumor cell lines were purchased from ATCC and cultured as recommended by the ATCC. RNA was extracted using the RNeasy kit (QIAGEN) and subjected to RT-PCR to amplify the eIF4E-specific RNA. The human eIF4E RNA signal was detected by Taqman RT-PCR according to the manufacturer's standard protocol (Applied Biosystems) using the following primers: forward primer, 5'-TGGCGACTGTCGAACCG-3'; reverse primer, 5'-AGATTCCGTTTTCTCCTCTTCTGTAG-3'; probe, 5'-FAM-AAACCACCCCTACTCCTAATCCCCCG-TAMRA-3'. The murine eIF4E RNA signal was detected by Taqman RT-PCR with the following primers: forward primer, 5'-AGGACGGTGGCTGATCACA-3'; reverse primer, 5'-TCTCTAGCCAGAAGCGATCGA-3'; probe, 5'-FAM-TGAACAAGCAGCAGAGACGGAGTGA-TAMRA-3'. These RT-PCR signals were normalized to total RNA using RiboGreen (Invitrogen). Proteins were isolated from cells, and Western blots were performed as described in ref. 40 using the following antibodies: eIF4E 1:500 (BD Biosciences); $\beta$-actin 1:10,000 (SigmaAldrich); survivin 1:200 (Millipore); and cyclin D1 1:500, VEGF 1:100, Bcl-2 1:500, and c-myc 1:200 (Santa Cruz Biotechnology Inc.). The eIF4E ELISA was run in a 96-well format using ELISA plates (Greiner Bio-One) coated overnight with $1.25 \mu \mathrm{g} / \mathrm{ml}$ donkey anti-mouse IgG in phosphate-buffered saline (Jackson Immunoresearch Laboratories Inc.). The next day, plates were washed 3 times in wash buffer (PBS, 0.05\% Tween 20) and blocked with $120 \mu \mathrm{l}$ Superblock blocking buffer (Pierce Biotechnology). Plates were incubated 1 hour at room temperature with the eIF4E antibody (BD Biosciences), then with lysate for 1 hour followed by a second eIF4E antibody for 1 hour (Cell Signaling Technologies), and finally in HRPO-linked antirabbit IgG (Amersham). Samples were washed 3 times with wash buffer in between each step. Signal was detected using the SuperSignal ELISA Femto maximum sensitivity substrate (Pierce Biotechnology) and read at $450 \mathrm{nM}$. The GAPDH ELISA was executed according to the manufacturer's protocol (Active Motif).

Protein translation studies. MDA-MB-231 breast cancer cells $(125,000)$ were seeded per well in a 6-well plate and transfected the next day $(3 \mu \mathrm{g} / \mathrm{ml}$ oligofectamine or lipofectamine/100 nM ASO). eIF4E reduction was assessed by quantitative RT-PCR from parallel samples 72 hours after transfection. Cells were washed twice with $1 \mathrm{ml}$ warm DMEM lacking methionine and cysteine plus $10 \%$ dialyzed FBS, then incubated in $4 \mathrm{ml}$ warm labeling medium for 20 minutes 72 hours after transfection. ${ }^{35} \mathrm{~S}$-methione/cysteine stock (Pro-Mix L-[35-S] in vitro cell labeling mix, $14.3 \mathrm{mCi} / \mathrm{ml}$, catalog no. SJQ0079; Amersham Biosciences) was diluted 1:250 in warm labeling medium (more than $\sim 0.06 \mathrm{mCi} / \mathrm{ml}$ final). Labeling medium $(1 \mathrm{ml}$ ) was added to each well for 1 hour at $37^{\circ} \mathrm{C}, 5 \% \mathrm{CO}_{2}$. Media were removed, and plates were washed twice with $2 \mathrm{ml}$ ice-cold $\left(4^{\circ} \mathrm{C}\right) \mathrm{PBS}$. We added $100 \mu \mathrm{l}$ $2 \%$ SDS, 100 mM Tris, pH 6.8, 5\% glycerol, and cells were scraped, put on ice and frozen at $-80^{\circ} \mathrm{C}$, and passed though a 22.5 -gauge needle. Lysate $(5 \mu \mathrm{l})$ was reserved for a protein concentration assay, and $5 \mathrm{mg}$ total protein/lane was added and electrophoresed through a $4 \%-12 \%$ NuPAGE gradient gel (Invitrogen) and dried on Whatman paper at $75^{\circ} \mathrm{C}$ for 3 hours. Radiography was quantitated per lane by phosphorimager after overnight exposure on a phosphor screen. As a control, samples were pretreated with cycloheximide $(75 \mu \mathrm{g} / \mathrm{ml})$ before the addition of label-to-block translation. For quantitation, the values for ${ }^{35} \mathrm{~S}$ incorporation were normalized to total RNA (RiboGreen; Invitrogen).

Endothelial cell tube formation assays. HUVECs were purchased from Cascade Biologics and cultured in Media 200 (Cascade Biologics). Transfections were executed using either lipofectin or oligofectamine as per the manufacturer's protocol (Invitrogen). HUVEC cells were plated on growth factor-depleted Matrigel (BD Biosciences) to elicit tube formation 52 hours after transfection (37). After 16 hours incubation, tube formation was evaluated semi-quantitatively by eye on a scale of 1 to 5 .

Alternately, HUVECs were cocultured with normal human dermal fibroblasts (38) in the recommended culture media and maintained according to the manufacturer's instructions (Cambrex). To ensure that the response of HUVEC cells did not simply reflect reduced cell number after transfection with 4E-ASO4, HUVECs were harvested from the mismatch or 4E-ASO 4 transfections 48 hours after transfection and counted with a hemocytometer. Viable cells (as determined by trypan blue exclusion) were then replated at 1,800 HUVECs per well on a bed of dermal fibroblasts (that had been plated 24 hours earlier in optimized media in 96-well plates; TCS Cellworks). Media were replenished every 2 or 3 days. Six days after HUVEC plating, cells were fixed with $70 \%$ cold ethanol and processed for anti-CD31/Alexa Fluor 488 immunofluorescence. Nuclear staining was performed using Hoechst dye (Invitrogen). Immunofluorescence was read by ArrayScan Vti, and the multi-parameter tube formation bioapplication was used for quantification (Cellomics). Data are presented as mean tube area (length $\times$ width of the CD31-positive structures) \pm SEM.

Animal studies. Xenograft studies were executed essentially as described in ref. 43 , with tumor-bearing mice randomized to treatment groups when group mean tumor volumes reached approximately $100 \mathrm{~mm}^{3}$. All mice were treated with an initial loading dose of $50 \mathrm{mg} / \mathrm{kg}$ ASO (either the eIF4E ASO or the mismatch ASO), on Monday, Tuesday, and Wednesday, followed by the indicated maintenance doses $(5-50 \mathrm{mg} / \mathrm{kg})$ beginning Friday and thrice weekly thereafter. Body weight was monitored each time tumors were measured. Tumors and livers were harvested as described in ref. 43 to evaluate eIF4E expression. All animal work was performed in an Association for Assessment of Laboratory Animal Care-certified facility and was approved by the Eli Lilly and Company Institutional Animal Care and Use Committee.

To evaluate the effects of reducing eIF4E in normal mouse tissues, mice were dosed twice weekly with $40 \mathrm{mg} / \mathrm{kg}$ ASO $(80 \mathrm{mg} / \mathrm{kg} / \mathrm{wk}$ compared with $75 \mathrm{mg} / \mathrm{kg} / \mathrm{wk}$ of the standard dosing in the PC-3 tumor efficacy studies). After 3 weeks mice were weighed and killed by cardiac puncture to obtain plasma. Plasma was shipped to LabCorp for determination of liver transaminase levels (alanine aminotransferase and aspartate aminotransferase). Liver and spleen weights were recorded.

High-content image-based analysis of activated caspase- 3 and TUNEL staining. MDA-MB-231 cells were transfected as described above for 72 hours. Cells were fixed with 3.7\% formaldehyde and washed in Dulbecco's PBS (D-PBS). Cells were permeabilized with $0.1 \%$ Triton X-100 in D-PBS, washed, and blocked in D-PBS containing 1\% BSA. Cells were then incubated for 1 hour with rabbit anti-activated caspase- 3 polyclonal antibody (BD Biosciences) diluted in D-PBS with 1\% BSA. Cells were washed 2 times with D-PBS then 
incubated for 1 hour with Alexa Fluor 488-conjugated goat anti-rabbit IgG (Invitrogen) and $200 \mathrm{ng} / \mathrm{ml}$ Hoechst 33342 (Invitrogen) diluted in D-PBS with $1 \%$ BSA. TUNEL staining was performed with the In Situ Cell Death Detection Kit, TMR Red (Roche Applied Science) as per the manufacturer's protocol. Stained plates were scanned using ArrayScan Vti (Cellomics). Quantitation of TUNEL and activated caspase-3 staining was performed using the Cellomic Target Activation Bioapplication with a minimum percent positive based on the control population for each cell line.

Immunohistochemical analyses. Tumors and livers from treated mice were formalin fixed and paraffin embedded. Antigen retrieval and antibody staining were performed on $5-\mu \mathrm{m}$ histological sections of the formalin-fixed, paraffin-embedded tumor and liver specimens essentially as described in ref. 44. eIF4E immunoreactivity was executed using the DAKO Animal Research Kit (Dako) and the eIF4E antibody (BD Biosciences) diluted 1:150 in DAKO antibody diluent. Adjacent sections from each specimen were incubated in a DAKO autostainer at room temperature for 5 minutes with $0.03 \%$ hydrogen peroxide containing sodium azide, rinsed in water and then in Tris-buffered saline Tween-20 (TBS-T), then incubated for 1 hour with either biotinylated eIF4E antibody or biotinylated mouse IgG2b at an equivalent protein concentration (1.67 $\mu \mathrm{g} / \mathrm{ml}$ ). Sections were rinsed twice with TBS-T and incubated with streptavidin conjugated to horseradish peroxidase (in PBS) for 15 minutes. Staining was visualized using $3,3^{\prime}$-diaminobenzidine as described in ref. 44. The immunohistochemical evaluation of eIF4E was validated by analyzing formalin-fixed, paraffin-embedded human cancer cells transfected with the eIF4E ASO. A fraction of the transfected cell pellets was retained before fixation for ELISA and Western blot analyses. Using the same antibody, a substantial reduction in eIF4E expression was evident by ELISA and Western blotting, and correlated with a dramatic reduction in eIF4E immunohistochemical detection (data not shown).

VEGF immunostaining was detected using monoclonal mouse antiVEGF, clone VG1 (DAKO), which labels VEGF isoforms VEGF-121, VEGF-165, and VEGF-189, as described in ref. 45. vWF (factor VIIIrelated antigen) was detected using DAKO rabbit anti-human vWF, which also cross-reacts strongly with murine vWF. Antigen retrieval was achieved with Proteinase $\mathrm{K}$ according to the manufacturer's instructions (catalog no. S3020; DAKO). The DAKO EnVision-HRP (DAB) was used to detect immunoreactivity with a 1:1,000 dilution of vWF antibody, using rabbit IgG at an equivalent protein concentration $(3.1 \mu \mathrm{g} / \mathrm{ml})$ as a control for each specimen.
Ki-67 staining. Tissues were dewaxed and rehydrated through a graded series of alcohols to PBS. Antigen retrieval was performed with $10 \mathrm{mM}$ citrate buffer, $\mathrm{pH} 6.0$, for 20 minutes at $90^{\circ}-93^{\circ} \mathrm{C}$ followed by 20 minutes at room temperature. For the immunohistochemical detection of Ki-67 positivity, slides were incubated with a monoclonal mouse antibody (1:75 dilution, clone MIB-1; DAKO) for 1 hour, utilizing the DAKO Animal Research Kit and a DAKO autostainer. Mouse IgG1 (DAKO) served as the negative control. Sections were counterstained with methyl green. Sections of human tonsil and human small intestine were the positive controls.

TUNEL staining in xenografts. Tissues were dewaxed and rehydrated through a graded series of alcohols to PBS. Apoptosis was detected using the ApoTag kit (Millipore) according to the manufacturer's directions. Sections of human tonsil and human small intestine, as well as a control tissue provided with the ApoTag kit, were the positive controls.

Quantification of Ki-67 and TUNEL stain. For each histologic section of xenografts from $4 \mathrm{E}-\mathrm{ASO}$ and mismatch control-treated mice, labeled cells were counted in each of 5 randomly chosen $\times 40$ microscopic fields (total magnification, $\times 400$ ). Counts from each of 2 separate staining runs for each stain were averaged for each specimen. Data are expressed as mean number of labeled cells per $\times 40$ field \pm SEM.

\section{Acknowledgments}

We wish to thank R. Gaynor, H. Pearce, J. McDonald, J. Starling, and J. Stille for their support and M. Marshall, C. Cohen, and L. Brail for helpful comments. We also wish to acknowledge Stephen G. Zimmer for providing invaluable guidance and support related to this work.

Received for publication March 7, 2007, and accepted in revised form June 27, 2007.

Address correspondence to: Jeremy R. Graff, Cancer Growth and Translational Genetics, Lilly Research Labs, Eli Lilly and Company, Lilly Corporate Center, DC 0546, Indianapolis, Indiana 46285, USA. Phone: (317) 277-0220; Fax: (317) 277-3652; E-mail: graff_jeremy@lilly.com.

Jeremy R. Graff, Bruce W. Konicek, Thomas M. Vincent, Rebecca L. Lynch, Julia H. Carter, and Eric G. Marcusson contributed equally to this work.
1. De Benedetti, A., and Graff, J.R. 2004. eIF4E expression and its role in malignancies and metastases. Oncogene. 23:3189-3199.

2. Mamane, Y., et al. 2004. eIF4E - from translation to transformation. Oncogene. 23:3172-3179.

3. Brunn, G.J., et al. 1997. Phosphorylation of the translational repressor PHAS-1 by the mammalian target of rapamycin. Science. 277:99-101.

4. Gingras, A.C., Kennedy, S.G., O'Leary, M.A., Sonenberg, N., and Hay, N. 1998. 4E-BP1, a repressor of the mRNA translation, is phosphorylated and inactivated by the Akt (PKB) signaling pathway. Genes Dev. 12:502-513.

5. Bjornsti, M.A., and Houghton, P.J. 2004. The TOR pathway: a target for cancer therapy. Nat. Rev. Cancer. 4:335-348.

6. Avdulov, S., et al. 2004. Activation of translation complex eIF4F is essential for the genesis and maintenance of the malignant phenotype in human mammary epithelial cells. Cancer Cell. 5:553-563.

7. Culjkovic, B., Topisirovic, I., Skrabanek, L., RuizGutierrez, M., and Borden, K.L. 2006. eIF4E is a central node of an RNA regulon that governs cellular proliferation. J. Cell Biol. 175:415-426.

8. Rosenwald, E.B., et al. 1995. Eukaryotic translation initiation factor $4 \mathrm{E}$ regulates expression of cyclin $\mathrm{D}$ at transcriptional and posttranscriptional levels. J. Biol. Chem. 270:21176-21180.

9. Rosenwald, I.B., Karatzas, A., Sonenberg, N., and Schmidt, E.V. 1993. Elevated levels of cyclin D1 protein in response to increased expression of eukaryotic initiation factor eIF4E. Mol. Cell. Biol. 13:7358-7363.

10. Rousseau, D., Kaspar, R., Rosenwald, I., Gehrke, L., and Sonenberg, N. 1996. Translation initiation of ornithine decarboxylase and nucleocytoplasmic transport of cyclin D1 mRNA are increased in cells overexpressing eukaryotic initiation factor 4E. Proc. Natl. Acad. Sci. U. S. A. 93:1065-1070.

11. Rosenwald, E.B., et al. 1995. Eukaryotic translation initiation factor $4 \mathrm{E}$ regulates expression of cyclin $\mathrm{D}$ at transcriptional and posttranscriptional levels. J. Biol. Chem. 270:21176-21180.

12. Rajasekhar, V.K., and Holland, E.C. 2004. Postgenomic global analysis of translational control induced by oncogenic signaling. Oncogene. 23:3248-3264.

13. De Benedetti, A., Joshi, B., Graff, J.R., and Zimmer, S.G. 1994. CHO cells transformed by the translation factor eIF4E display increased c-myc expression, but require overexpression of Max for tumori- genicity. Mol. Cell. Differ. 2:347-371.

14. Lazaris-Karatzas, A., Montine, K.S., and Sonenberg, N. 1990. Malignant transformation by a eukaryotic initiation factor subunit that binds to mRNA cap. Nature. 345:544-547.

15. Zimmer, S.G., DeBenedetti, A., and Graff, J.R. 2000. Translational control of malignancy: the mRNA cap-binding protein, eIF4E, as a central regulator of tumor formation, growth, invasion and metastasis. Anticancer Res. 20:1343-1351.

16. Shantz, L.M., and Pegg, A.E. 1994. Overproduction of ornithine decarboxylase caused by relief of translational repression is associated with neoplastic transformation. Cancer Res. 54:2313-2316.

17. Lazaris-Karatzas, A., et al. 1992. Ras mediates translation initiation factor 4E-induced malignant transformation. Genes Dev. 6:1631-1642.

18. Ruggero, D., et al. 2004. The translation factor eIF4E promotes tumor formation and cooperates with c-Myc in lymphomagenesis. Nat. Med. 10:484-486.

19. Wendel, H.G., et al. 2004. Survival signalling by Akt and eIF4E in oncogenesis and cancer therapy. Nature. 428:332-337.

20. Rinker-Schaeffer, C.W., Graff, J.R., DeBenedetti, A., Zimmer, S.G., and Rhoads, R.E. 1993. Decreasing 
the level of translation initiation factor $4 \mathrm{E}$ with antisense RNA causes reversal of ras-mediated transformation and tumorigenesis of cloned rat embryo fibroblasts. Int. J. Cancer. 55:841-847.

21. Graff, J.R., Boghaert, E.R., DeBenedetti, A., Tudor, D.M., and Zimmer, S.G. 1995. Reduction of translation initiation factor $4 \mathrm{E}$ reduces tumor growth, invasion and metastasis of ras-transformed cloned rat embryo fibroblast. Int. J. Cancer. 60:255-263.

22. Nathan, C.A., et al. 1997. Elevated expression of eIF4E and FGF-2 isoforms during vascularization of breast carcinomas. Oncogene. 15:1087-1095.

23. DeFatta, R.J., Nathan, C.A., and DeBenedetti, A. 2000. Antisense RNA to eIF4E suppresses oncogenic properties of a head and neck squamous cell carcinoma cell line. Laryngoscope. 110:928-933.

24. Graff, J.R., et al. 1997. Translation of ODC mRNA and polyamine transport are suppressed in rastransformed CREF cells by depleting translation initiation factor 4E. Biochem. Biophys. Res. Commun. 240:15-20.

25. Rousseau, D., Gingras, A.C., Pause, A., and Sonenberg, N. 1996. The eIF4E-binding proteins 1 and 2 are negative regulators of cell growth. Oncogene. 13:2415-2420.

26. Zhang, H., et al. 2000. Reduction of liver Fas expression by an antisense oligonucleotide protects mice from fulminant hepatitis. Nat. Biotech nol. 18:862-867.

27. Geary, R.S., et al. 2001. Pharmacokinetic properties of 2'-O-(2-methoxyethyl)-modified oligonucleotide analogs in rats. J. Pharmacol. Exp. Ther. 296:890-897.

28. Dean, N.M., and Bennett, C.F. 2003. Antisense oli- gonucleotide-based therapeutics for cancer. Oncogene. 22:9087-9096.

29. Yu, R.Z., et al. 2004. Tissue disposition of 2'-O(2-methoxy) ethyl modified antisense oligonucleotides in monkeys. J. Pharm. Sci. 93:48-59.

30. Mamane, Y., et al. 2007. Epigenetic activation of a subset of mRNAs by eIF4E explains its effects on cell proliferation. PLOS ONE. 2:e242.

31. Larsson, O., et al. 2006. Apoptosis resistance downstream of eIF4E: posttranscriptional activation of an anti-apoptotic transcript carrying a consensus hairpin structure. Nucleic Acids Res. 34:4375-4386.

32. Herbert, T.P., Fahraeus, R., Prescott, A., Lane, D.P., and Proud, C.G. 2000. Rapid induction of apoptosis mediated by peptides that bind initiation factor 4E. Curr. Biol. 10:793-796.

33. Li, S., et al. 2004. Translation initiation factor 4E blocks endoplasmic reticulum-mediated apoptosis. J. Biol. Chem. 279:21312-21317.

34. Li, S., et al. 2003. Translation factor eIF4E rescues cells from Myc-dependent apoptosis by inhibiting cytochrome c release. J. Biol. Chem. 278:3015-3022.

35. Polunovsky, V.A et al. 1996. Translational control of programmed cell death: eukaryotic translation initiation factor 4E blocks apoptosis in growthfactor-restricted fibroblasts with physiologically expressed or deregulated Myc. Mol. Cell. Biol. 16:6573-6581

36. Jiang, B.H., Zheng, J.Z., Aoki, M., and Vogt, P.K. 2000. Phosphatidylinositol 3-kinase signaling mediates angiogenesis and expression of vascular endothelial growth factor in endothelial cells. Proc. Natl. Acad. Sci. U. S. A. 97:1749-1753.
37. Jackson, C.J., and Jenkins, K.L. 1991. Type I collagen fibrils promote rapid vascular tube formation upon contact with the apical side of cultured endothelium. Exp. Cell Res. 192:319-323.

38. Bishop, E.T., et al. 1999. An in vitro model of angiogenesis: basic features. Angiogenesis. 3:335-344.

39. Chi, K.N., et al. 2005. A phase I pharmacokinetic and pharmacodynamic study of OGX-011, a 2'methoxyethyl antisense oligonucleotide to clusterin, in patients with localized prostate cancer. J. Natl. Cancer Inst. 97:1287-1296.

40. Graff, J.R., et al. 2000. Increased AKT activity contributes to prostate cancer progression by dramatically accelerating prostate tumor growth and diminishing p27Kip1 expression. J. Biol. Chem. 275:24500-24505.

41. Majumder, P.K., and Sellers, W.R. 2005. Aktregulated pathways in prostate cancer. Oncogene. 24:7465-7474.

42. St. Croix, B., et al. 2000. Genes expressed in human tumor endothelium. Science. 289:1197-1202.

43. Graff, J.R., et al. 2005. The protein kinase C $\beta$ selective inhibitor, Enzastaurin (LY317615.HCl), suppresses signaling through the AKT pathway, induces apoptosis, and suppresses growth of human colon cancer and glioblastoma xenografts. Cancer Res. 65:7462-7469.

44. Carter, J.H., et al. 2004. Pak-1 expression increases with progression of colorectal carcinomas to metastasis. Clin. Cancer Res. 10:3448-3456.

45. Turley, H., et al. 1998. Expression of VEGF in routinely fixed material using a new monoclonal antibody VG1. J. Pathol. 186:313-318. 